\section{Genotype-phenotype correlations in tuberous sclerosis}

EDITOR-Van Slegtenhorst et al ${ }^{1}$ reported a mutational analysis of 225 patients with tuberous sclerosis (TSC) and concluded that there was no evidence for genotypephenotype correlation. At virtually the same time, Jones et $a l^{2}$ from Cardiff published supportive evidence for genotype-phenotypic correlation in their comprehensive mutational analysis of TSC1 and TSC2 in 150 families. How can such conflicting conclusions be reconciled?

It is now well established that tuberous sclerosis (TSC) is a multisystem disorder characterised by an autosomal dominant pattern of inheritance, locus heterogeneity (TSC1 on 9q34 and TSC2 on 16p13.3), and widely variable expression. It is associated with neurodevelopmental abnormalities, manifestations of which include epilepsy, mental retardation, and behavioural syndromes such as autism. Both genes have been positionally cloned and act as tumour suppressors. Linkage analysis suggested that about half of large families were linked to TSC1 and half to TSC2. It was assumed that the same would apply to sporadic cases (representing about $70 \%$ of all TSC cases).

Jones et $a l^{3}$ published the first findings of differences between TSC1 and TSC2 associated familial and sporadic TSC. They found an under-representation of TSC1 and an over-representation of TSC 2 in sporadic cases. They proceeded to compare rates of mental retardation (defined as "present when formal developmental assessment had revealed an overall developmental quotient under 70, when unassisted mainstream schooling was impossible because of learning difficulties (not behavioural problems) or where an adult was institutionalised or required supervision in the community") among patients with TSC1 versus TSC2 mutations and found TSC1 cases to have lower rates of mental retardation. In their recent paper, ${ }^{2}$ they reported mutation analysis of TSC 1 and TSC 2 in $80 \%$ of 150 cases, and again found TSC1 in fewer than expected cases in the sporadic group and more "intellectual disability" in TSC2 cases owing to sporadic mutations. They argue for a difference in severity of the TSC1 and TSC2 associated disorder.

In contrast, van Slegtenhorst et al ${ }^{1}$ screened for TSC1 mutations in 225 unrelated patients and found 29 mutations (16\% of familial cases and $11 \%$ of sporadic cases). Patients were then scored for the "most frequent skin, brain, kidney, and heart lesions detected in TSC", and they found no genotype-phenotype correlation between truncating mutations and in frame deletions in exons 7 and 15.

It is clear that the Cardiff group were examining the concept of genotype-phenotype differences in severity between TSC1 and TSC2, using mental retardation as the yardstick, while the Rotterdam team were searching for physical phenotype-genotype correlation, comparing the presence or absence of physical features with different mutational types. It is not surprising therefore that their conclusions appeared superficially contradictory.
We recently conducted a postal survey of all members of the Tuberous Sclerosis Association of Great Britain with affected children ( $n=995)$. The questionnaire survey asked parents to endorse the presence/absence of physical features of TSC, developmental problems, and the lifetime presence/absence of behavioural, psychological, and psychiatric problems. A total of 510 families (51.3\%) responded to the survey. Based on symptoms and signs reported in parents and children, we were able to divide the sample into 404 sporadic and 69 familial cases; 37 cases were excluded because of lack of information or when subjects did not meet diagnostic criteria for TSC. In order to ensure a probandwise comparison of subjects, seven further cases were excluded where a parent had been diagnosed with TSC before the birth of a child. Using the familial-sporadic design "by proxy" to (arguably) compare TSC1 and TSC2, we found no difference in the rates of any of the physical features of TSC or presence/absence of reported brain abnormalities (cortical tubers or subependymal nodules). In the probandwise comparison of the rates of mental retardation, we did, however, find that significantly more mental retardation was reported in sporadic versus familial cases $\left(65 \% v 46 \%, \chi^{2}=10.14, \mathrm{df}=1\right.$, $\mathrm{p}=0.0015)$. This suggests an odds ratio of mental retardation in sporadic cases of 2.44 (1.98/0.81).

Our survey findings are therefore consistent with the work of both van Slegtenhorst et $a l^{1}$ and Jones et $a l^{2}$ and suggest that TSC1/TSC2 cases may differ in severity and number of lesions, rather than the nature and quality of the manifestation. The likelihood of second hits may, for instance, differ in the two forms, accounting for the observed differences.

It does seem as though TSC1 and TSC2 do not represent distinct clinical syndromes (akin to neurofibromatosis type 1 and type 2, for instance) based on the presence/absence of certain core features. The likelihood that differences will be based on severity or more subtle manifestations highlights the importance of refining research approaches in search of these genotype-phenotype correlations. Mental retardation should be quantified in a more standardised way and physical features documented in more detail. Moreover, careful attention will need to be paid to the modes of ascertainment when comparing TSC1 and TSC2 cases.

PETRUS J DE VRIES PATRICK F BOLTON

Developmental Psychiatry Section, University of Cambridge, Douglas House, $18 b$ Trumpington Road, Cambridge CB2 $2 A H$, UK

Correspondence to: Drde Vries,pd215@cam.ac.uk

1 van Slegtenhorst $M$, Verhoef $S$, Tempelaars $A$, et al. Mutational spectrum of the TSC1 gene in a cohort of 225 tuberous sclerosis complex patients: no evidence for genotype-phenotype correlation. F Med Genet 1999;36:285-9.

2 Jones AC, Shyamsundar MM, Thomas MW, et al. Comprehensive mutation analysis of TSC 1 and TSC 2 - and phenotypic correlations in 150 families with tuberous sclerosis. Am f Hum Genet 1999;64:1305-15.

3 Jones AC, Daniells CE, Snell RG, et al. Molecular genetic and phenotypic analysis reveals differences between TSC1 and TSC2 associated familial and sporadic tuberous sclerosis. Hum Mol Genet 1997;6:2155-61. 